\title{
Stability of a system with cosmic rays and waves
}

\author{
Y.-Y. Lo ${ }^{1}$ and C.-M. Ko ${ }^{2}$ \\ 1 Institute of Astronomy, National Central University, Chung-Li, Taiwan 320, R.O.C. \\ e-mail: d909002@astro.ncu.edu.tw \\ 2 Institute of Astronomy, Department of Physics and Center for Complex Systems, National Central University, Chung-Li, \\ Taiwan 320, R.O.C. \\ e-mail: cmko@astro.ncu.edu.tw
}

Received 15 December 2006 / Accepted 27 March 2007

ABSTRACT

\begin{abstract}
Aims. We investigate the the stability of a cosmic-ray plasma system using the hydrodynamic approach. Methods. The system we are considering comprises cosmic rays and two oppositely propagating Alfvén waves. The thermal plasma flow is treated as a background, such that the feedback of the cosmic rays and waves is neglected. Steady-state solutions exist for the system, and when two waves are present, the steady state is non-uniform. Linear stability analysis on the steady-state solutions shows that for short wavelengths, large cosmic ray energy density, and small wave energy density the system is stable.

Results. We present in detail the case of uniform background flow. The system is most unstable in the upstream region and becomes stable in the downstream region in the linear regime for short-enough wavelengths. We also speculate on the implication of the instability.
\end{abstract}

Key words. ISM: cosmic rays - magnetohydrodynamics (MHD) - instability - waves

\section{Introduction}

Cosmic rays are high energy charged particles which permeate through the galaxy. In most astrophysical applications collisions between cosmic rays and thermal plasma are negligible, and the interaction of the cosmic rays and thermal plasma is mediated via the embedded magnetic field. Cosmic rays interact and are scattered by hydromagnetic waves or irregularities (e.g., gyroresonant scattering). As a result, cosmic rays advect with the plasma flow and diffuse through the plasma as well. Moreover, when waves of different phase velocities are present, cosmic rays undergo stochastic acceleration (or so-called second-order Fermi process) and diffuse in momentum space. As cosmic rays propagate through the plasma, they excite hydromagnetic waves (e.g., Alfvén waves) via cosmic-ray streaming instability. These waves in turn serve as the scattering centres for the cosmic rays. Thus the interaction can be described in a self-consistent manner. The couplings between cosmic rays and thermal plasma depend on the collision frequency of the waves and cosmic rays, which depends in part on the wave amplitude. The upshot is advection, spatial and momentum diffusion of cosmic rays all depend on the energy density of waves. For cosmic-ray propagation the reader is referred to, e.g., (Skilling 1975a,b; Schlickeiser 1989, 2002). An important application of cosmic-ray propagation is cosmic ray acceleration (see e.g., reveiws by Drury 1983; Blandford \& Eichler 1987; Berezhko \& Krymskii 1988; Jones \& Ellison 1991).

When the energy densities of the cosmic rays and waves are comparable to the thermal plasma, their influence or backreaction on the thermal plasma cannot be ignored, taking into proper account the momentum and energy exchanges between thermal plasma flow and cosmic rays and waves, we see that we are dealing with a complicated cosmic-ray-plasma system. There are three approaches to the problem.
(A) The ultimate approach is the kinetic theory approach in which the plasma and cosmic rays are described by the same phase-space distribution function and the waves are described by the collective behaviour of the magnetic field.

(B) The intermediate approach is the hybrid approach in which the thermal plasma is described as a fluid, the cosmic ray is described by a phase-space distribution function, and the waves are described by power spectra.

(C) The simplest approach is the hydrodynamic approach in which thermal plasma, cosmic rays and waves are described by different interacting fluids.

Although the hydrodynamic approach cannot provide the spectra of cosmic rays and waves, it is still a good approximation to use to study the structure and dynamics of the system. The hydrodynamic approach is very useful and successful in the investigation of cosmic-ray-modified shocks (hydrodynamic shocks modified by the feedback of cosmic rays) without considering waves explicitly (e.g., Drury \& Völk 1981; Axford et al. 1982; Webb et al. 1986; Webb 1987; Ko et al. 1997), with one Alfvén wave (e.g., McKenzie \& Völk 1982), and two oppositely-propagating Alfvén waves (Ko 2001).

Ten years ago, Malkov (1997a,b) pointed out that the hydrodynamic approach is a singular limiting case of the hybrid approach. This gives rise to some of the shortcomings mentioned in various studies (e.g., Heavens 1984; Achterberg et al. 1984; Jones \& Kang 1990; Jones \& Ellison 1991; Ko 1995). Bearing these limitations in mind, we adopt this approach in this paper for its simplicity.

The stability of the system in this approach has been addressed in different situations. If one neglects waves in the system, a uniform background state is unconditionally stable (e.g., Ptuskin 1981). For the case of non-uniform background (such as cosmic-ray modified shocks), the system is susceptible to the so-called squeezing instability (e.g., Drury \& Falle 1986; 
Zank \& McKenzie 1987; Zank et al. 1990; Kang et al. 1992; Zank et al. 1993). If gravity (external or self) is included in the system, the Parker or Parker-Jeans instability can be modified qualitatively by cosmic rays (e.g., Kuznetsov \& Ptuskin 1983; Ryu et al. 2003; Hanasz \& Lesch 2003; Hanasz et al. 2004; Kuwabara et al. 2004; Kuwabara \& Ko 2006).

The stability of the uni-directional wave system (i.e., only one self-excited Alfvén wave) was studied before (e.g., McKenzie \& Webb 1984; Zank 1989; Ko et al. 1991; Ko 1991; Ko \& Jeng 1994). The stability of a uniform background was analysed thoroughly, (McKenzie \& Webb 1984; Zank 1989; Ko \& Jeng 1994), and the result is that the system is susceptible to slow magneto-acoustic perturbations. To our knowledge the stability of a system with both forward and backward Alfvén waves has not been considered. Although Ko \& Jeng (1994) allowed for both waves in their perturbation analysis, their background state was still uni-directional. In this paper we study a simpler system but still with two Alfvén waves. We focus on the interactions between cosmic rays and waves, and consider the thermal plasma flow as a reservoir of momentum and energy (i.e., as a prescribed background, Jiang et al. 1996). In our model the interactions (e.g., stochastic acceleration, diffusion) depend on the energy density of the waves, the detail is described in Sect. 2, and we present the steady state solutions to this model in Sect. 3. In Sect. 4 we present the standard linear stability analysis. Section 5 works out the stable and unstable regions. Section 6 provides a summary and discussion.

\section{The model}

Cosmic rays interact with plasma via magnetic irregularities or waves, and waves can be generated by the cosmic rays streaming instability. The system can be described self-consistently by a four-fluid model, which consists of thermal plasma, cosmic rays and two oppositely propagating Alfvén waves (Ko 1992). The cosmic rays and waves are considered as massless fluids and are described by corresponding energy densities or pressures. In this work, we adopted a reduced system in which the plasma flow (including the mean magnetic field) is treated as a background (or a reservoir of momentum and energy) (Jiang et al. 1996). The reduced system is governed by the cosmic ray and wave energy equations. In a given plasma flow and mean magnetic field they are,

$\frac{\partial E_{\mathrm{c}}}{\partial t}+\nabla \cdot F_{\mathrm{c}}=\left[\boldsymbol{U}+\left(e_{+}-e_{-}\right) V_{\mathrm{A}}\right] \cdot \nabla P_{\mathrm{c}}+\frac{P_{\mathrm{c}}}{\tau}$,

$\frac{\partial E_{\mathrm{w}}^{ \pm}}{\partial t}+\nabla \cdot F_{\mathrm{w}}^{ \pm}=\boldsymbol{U} \cdot \nabla P_{\mathrm{w}}^{ \pm} \mp e_{ \pm} V_{\mathrm{A}} \cdot \nabla P_{\mathrm{c}}-\frac{P_{\mathrm{c}}}{2 \tau}$,

where \pm corresponds to forward and backward propagations along the mean magnetic field, $\boldsymbol{U}$ and $V_{\mathrm{A}}$ are the flow velocity and Alfvén velocity. The energies and pressures are related by the polytropic relations,

$E_{\mathrm{c}}=\frac{P_{\mathrm{c}}}{\left(\gamma_{\mathrm{c}}-1\right)}, \quad E_{\mathrm{w}}^{ \pm}=2 P_{\mathrm{w}}^{ \pm}$,

and the cosmic ray and wave energy fluxes are defined as

$F_{\mathrm{c}}=\left(E_{\mathrm{c}}+P_{\mathrm{c}}\right)\left[\boldsymbol{U}+\left(e_{+}-e_{-}\right) V_{\mathrm{A}}\right]-\overleftrightarrow{\kappa} \cdot \nabla E_{\mathrm{c}}$

$F_{\mathrm{w}}^{ \pm}=\left(\boldsymbol{U} \pm V_{\mathrm{A}}\right) E_{\mathrm{w}}^{ \pm}+\boldsymbol{U} P_{\mathrm{w}}^{ \pm}$.

The interactions among the fluids depend on the waves. Ko (Ko 1992) gave a simple model for the "fraction of wave speed" $e_{ \pm}$, the cosmic ray diffusion coefficient $\overleftrightarrow{\kappa}$, and the stochastic acceleration time scale $\tau$,

$$
\begin{aligned}
e_{ \pm} & =\frac{P_{\mathrm{w}}^{ \pm}}{\left(P_{\mathrm{w}}^{+}+P_{\mathrm{w}}^{-}\right)}, \quad \overleftrightarrow{\kappa}=\frac{a c^{2} \hat{\boldsymbol{B}} \hat{\boldsymbol{B}}}{3 \alpha\left(P_{\mathrm{w}}^{+}+P_{\mathrm{w}}^{-}\right)} \\
\frac{1}{\tau} & =16 b \alpha \frac{V_{\mathrm{A}}^{2}}{c^{2}} \frac{P_{\mathrm{w}}^{+} P_{\mathrm{w}}^{-}}{\left(P_{\mathrm{w}}^{+}+P_{\mathrm{w}}^{-}\right)}
\end{aligned}
$$

where $\hat{\boldsymbol{B}}$ is the unit vector of the magnetic field, $c$ is the speed of light, $\alpha$ represents the coupling, and $a \leq 1$ and $b \geq 1$ but $\frac{3}{4} \leq a b \leq 1$. For ultra-relativistic cosmic rays $a=b=1$ (and $\left.\gamma_{\mathrm{c}}=\frac{3}{4}\right)$. Note that $e_{+}+e_{-}=1$.

The energies of cosmic rays and waves can be changed via three mechanisms (see e.g., Ko 2001):

(a) work done by thermal plasma flow against pressure gradients $\left(\boldsymbol{U} \cdot \nabla P_{\mathrm{c}}\right.$ and $\boldsymbol{U} \cdot \nabla P_{\mathrm{w}}^{ \pm}$on the right hand side of Eqs. (1) and (2), respectively);

(b) cosmic-ray streaming instability $\left(\left(e_{+}-e_{-}\right) V_{\mathrm{A}} \cdot \nabla P_{\mathrm{c}}\right.$ and $e_{ \pm} V_{\mathrm{A}}$. $\nabla P_{\mathrm{c}}$ on the right hand side of Eqs. (1) and (2), respectively);

(c) stochastic acceleration or second-order Fermi process $\left(P_{\mathrm{c}} / \tau\right.$ and $P_{\mathrm{c}} / 2 \tau$ on the right-hand side of Eqs. (1) and (2), respectively).

Mechanisms (a) and (b) may increase or decrease the energy of cosmic rays, while mechanism (c) always increases the cosmic ray energy (and always decreases the wave energy). Mechanisms (b) and (c) do not involve interactions with the thermal plasma directly.

\section{Steady state solutions}

Jiang et al. (1996) studied the steady state of the system (the so-called nonlinear test particle picture). They found solutions in one-dimensional geometry with uniform flow and magnetic field, where the magnetic field is parallel to the flow direction. They expressed the steady-state solutions in terms of two integrals: the wave-action integral $W_{\mathrm{A}}$ and the streaming integral $S$, where

$$
\begin{aligned}
& W_{\mathrm{A}}=F_{\mathrm{c}}+\frac{\left(U+V_{\mathrm{A}}\right)^{2}}{V_{\mathrm{A}}} E_{\mathrm{w}}^{+}-\frac{\left(U-V_{\mathrm{A}}\right)^{2}}{V_{\mathrm{A}}} E_{\mathrm{w}}^{-}, \\
& S=V_{\mathrm{A}} P_{\mathrm{c}}+\left(U+V_{\mathrm{A}}\right) E_{\mathrm{w}}^{+}-\left(U-V_{\mathrm{A}}\right) E_{\mathrm{w}}^{-} .
\end{aligned}
$$

In terms of these integrals and a suitable length scale, the forward and backward waves are described by the following two ordinary differential equations,

$$
\begin{aligned}
& \frac{\mathrm{d} P_{\mathrm{w}}^{+}}{\mathrm{d} x}=-\frac{P_{\mathrm{w}}^{+}\left(D_{\mathrm{c}}+2 \beta P_{\mathrm{c}} P_{\mathrm{w}}^{-}\right)}{2\left(M_{\mathrm{A}}+1\right)\left(P_{\mathrm{w}}^{+}+P_{\mathrm{w}}^{-}\right)}, \\
& \frac{\mathrm{d} P_{\mathrm{w}}^{-}}{\mathrm{d} x}=\frac{P_{\mathrm{w}}^{-}\left(D_{\mathrm{c}}-2 \beta P_{\mathrm{c}} P_{\mathrm{w}}^{+}\right)}{2\left(M_{\mathrm{A}}-1\right)\left(P_{\mathrm{w}}^{+}+P_{\mathrm{w}}^{-}\right)},
\end{aligned}
$$

where $\beta=\frac{4}{3} a b\left(1 \leq \beta \leq \frac{4}{3}\right)$, the Alfvén Mach number $M_{\mathrm{A}}=$ $U / V_{\mathrm{A}}$, and the cosmic ray pressure, flux, and gradient are

$$
\begin{aligned}
& P_{\mathrm{c}}=S-2\left(M_{\mathrm{A}}+1\right) P_{\mathrm{w}}^{+}+2\left(M_{\mathrm{A}}-1\right) P_{\mathrm{w}}^{-}, \\
& F_{\mathrm{c}}=W_{\mathrm{A}}-2\left(M_{\mathrm{A}}+1\right)^{2} P_{\mathrm{w}}^{+}+2\left(M_{\mathrm{A}}-1\right)^{2} P_{\mathrm{w}}^{-},
\end{aligned}
$$

$$
\begin{aligned}
D_{\mathrm{c}}= & \gamma_{\mathrm{c}} P_{\mathrm{c}}\left[\left(M_{\mathrm{A}}+1\right) P_{\mathrm{w}}^{+}+\left(M_{\mathrm{A}}-1\right) P_{\mathrm{w}}^{-}\right] \\
& -\left(\gamma_{\mathrm{c}}-1\right)\left(P_{\mathrm{w}}^{+}+P_{\mathrm{w}}^{-}\right) F_{\mathrm{c}} .
\end{aligned}
$$



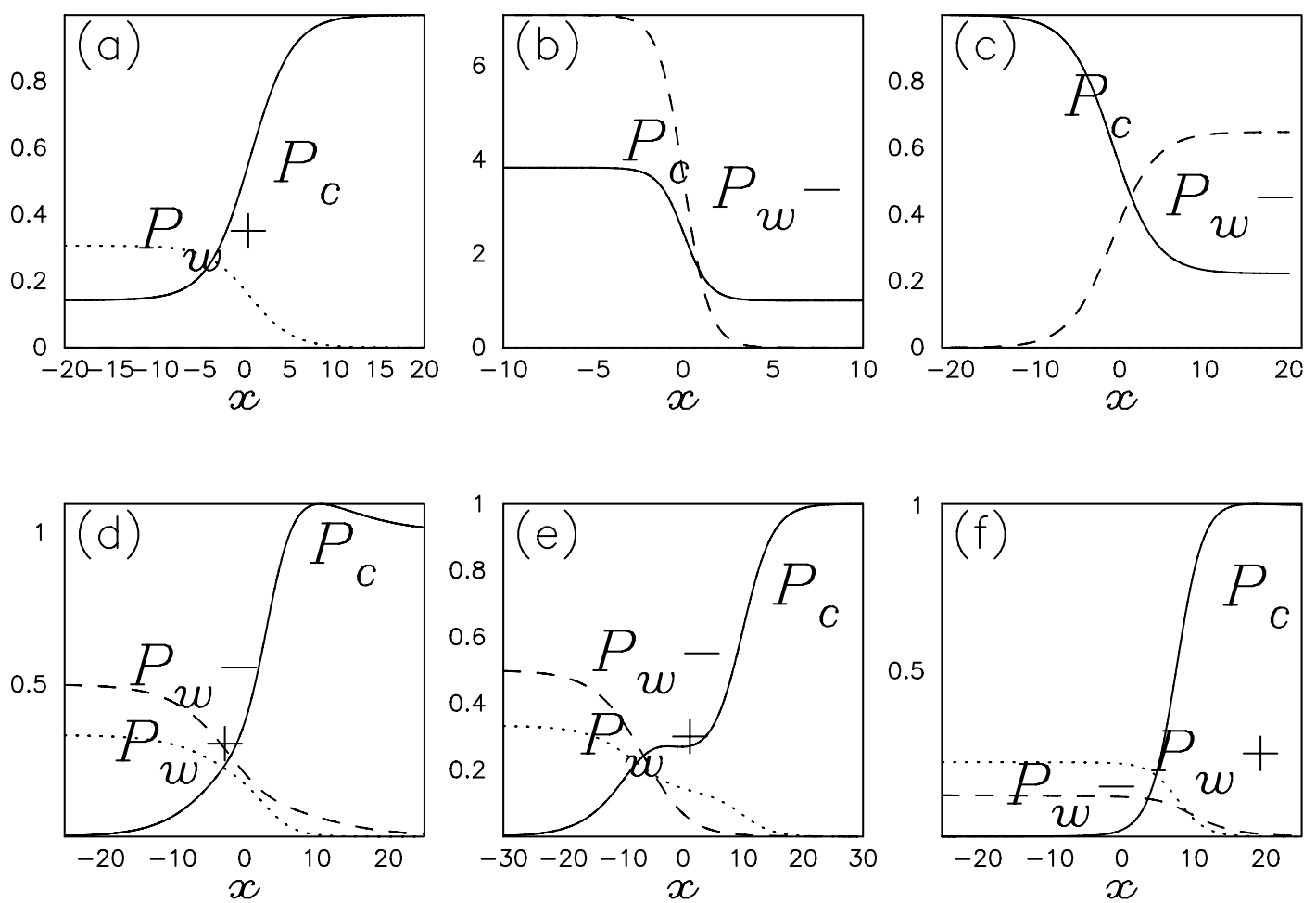

Fig. 1. The steady state profiles of cosmic ray and Alfvén wave pressures (Jiang et al. 1996). a)-c) are systems with one wave only (i.e., unidirectional wave systems), and d)-f) are systems with both waves (stochastic acceleration in operation). In these examples, $\gamma_{\mathrm{c}}=\frac{4}{3}, \beta=\frac{4}{3}$, and the Alfvénic Mach numbers are: a) $M_{\mathrm{A}}=0.4$, b) $M_{\mathrm{A}}=1.2$, c) $M_{\mathrm{A}}=0.4$, d) $M_{\mathrm{A}}=2.0$, e) $M_{\mathrm{A}}=2.0$, and f) $M_{A}=1.5$.

A solution is a physical solution if all of its pressures (or energy densities) are non-negative and finite, and it approaches a uniform state at both far upstream and downstream regions (i.e., $x \rightarrow \pm \infty)$. Typical physical solutions are shown in Fig. 1. The upper three panels are uni-directional wave systems (in which only one wave exists), and the lower three panels are two-wave systems. The wave pressures always decrease down the flow, while the cosmic ray pressure increases in general, but not necessarily monotonically.

Ko (1998) studied the same model but with shocked background, in which the upstream and downstream velocity $U_{1}$ and $U_{2}$ are prescribed constant. Beside the dependent of the parameters in Jiang et al. (1996), the behaviour of the cosmic ray and wave pressures depend on the compression ratio and the location of the shock. The compression ratio $q$ is defined as

$q=\frac{U_{1}}{U_{2}}=\left(\frac{V_{\mathrm{A} 1}}{V_{\mathrm{A} 2}}\right)^{2}$.

The location of the shock can be parameterized by the value of the pressure of waves just upstream of the shock, because the wave pressures upstream is monotonically decreasing down the flow. The location parameter $\sigma$ can be defined as the ratio of the wave pressure at shock to the wave pressure at far upstream. Figure 2 shows an example of results from shocked backgrounds. In this example, only a forward wave exists upstream of the shock, while both waves occur downstream because the shock can generate waves. The parameters used in this example are $M_{\mathrm{A} 1}=U_{1} / V_{\mathrm{A} 1}=2.0, q=1.5$ and $\sigma=0.6$.

\section{Linear stability analysis}

Although physical steady-state solutions exist for the system Eqs. (1)-(2), we want to understand if they are susceptible to small perturbations. We perform standard linear perturbation analysis on the system Eqs. (1)-(2). The small perturbations $\delta \psi=\left(\delta P_{\mathrm{c}}, \delta P_{\mathrm{w}}^{ \pm}\right)$satisfy the following linear equations (where $\psi=\left(P_{\mathrm{c}}, P_{\mathrm{w}}^{ \pm}\right)$is the unperturbed state) (cf., Ko \& Jeng 1994):

$$
\begin{aligned}
\frac{\partial \delta P_{\mathrm{c}}}{\partial t}+ & {\left[\boldsymbol{U}+\left(e_{+}-e_{-}\right) V_{\mathrm{A}}\right] \cdot \nabla \delta P_{\mathrm{c}} } \\
+ & 2 \gamma_{\mathrm{c}} P_{\mathrm{c}} \nabla \cdot\left[\frac{\left(e_{-} \delta P_{\mathrm{w}}^{+}-e_{+} \delta P_{\mathrm{w}}^{-}\right)}{\left(P_{\mathrm{w}}^{+}+P_{\mathrm{w}}^{-}\right)} V_{\mathrm{A}}\right] \\
& -\nabla \cdot\left[\frac{c^{2}}{3 \alpha\left(P_{\mathrm{w}}^{+}+P_{\mathrm{w}}^{-}\right)} \hat{\boldsymbol{B}} \hat{\boldsymbol{B}} \cdot \nabla \delta P_{\mathrm{c}}\right] \\
& -16 \alpha\left(\gamma_{\mathrm{c}}-1\right) \frac{V_{\mathrm{A}}^{2}}{c^{2}}\left[\left(e_{-}^{2} \delta P_{\mathrm{w}}^{+}+e_{+}^{2} \delta P_{\mathrm{w}}^{-}\right) P_{\mathrm{c}}+\delta P_{\mathrm{c}}\right] \\
+ & \frac{2\left(e_{-} \delta P_{\mathrm{w}}^{+}-e_{+} \delta P_{\mathrm{w}}^{-}\right)}{\left(P_{\mathrm{w}}^{+}+P_{\mathrm{w}}^{-}\right)} V_{\mathrm{A}} \cdot \nabla P_{\mathrm{c}} \\
+ & \gamma_{\mathrm{c}} \delta P_{\mathrm{c}} \nabla \cdot\left[\boldsymbol{U}+\left(e_{+}-e_{-}\right) V_{\mathrm{A}}\right] \\
+ & \nabla \cdot\left[\frac{c^{2}\left(\delta P_{\mathrm{w}}^{+}+\delta P_{\mathrm{w}}^{-}\right)}{3 \alpha\left(P_{\mathrm{w}}^{+}+P_{\mathrm{w}}^{-}\right)^{2}} \hat{\boldsymbol{B}} \hat{\boldsymbol{B}} \cdot \nabla P_{\mathrm{c}}\right] \\
& =0
\end{aligned}
$$

$$
\begin{aligned}
\frac{\partial \delta P_{\mathrm{w}}^{ \pm}}{\partial t}+ & \left(\boldsymbol{U} \pm V_{\mathrm{A}}\right) \cdot \nabla \delta P_{\mathrm{w}}^{ \pm}+ \pm \frac{1}{2} e_{ \pm} V_{\mathrm{A}} \cdot \nabla \delta P_{\mathrm{c}} \\
+ & 4 \alpha \frac{V_{\mathrm{A}}^{2}}{c^{2}}\left[\left(e_{-}^{2} \delta P_{\mathrm{w}}^{+}+e_{+}^{2} \delta P_{\mathrm{w}}^{-}\right) P_{\mathrm{c}}+\delta P_{\mathrm{c}}\right] \\
+ & \delta P_{\mathrm{w}}^{ \pm} \nabla \cdot\left(\frac{3}{2} \boldsymbol{U} \pm V_{\mathrm{A}}\right) \\
+ & \frac{\left(e_{-} \delta P_{\mathrm{w}}^{+}-e_{+} \delta P_{\mathrm{w}}^{-}\right)}{2\left(P_{\mathrm{w}}^{+}+P_{\mathrm{w}}^{-}\right)} V_{\mathrm{A}} \cdot \nabla P_{\mathrm{c}} \\
& =0
\end{aligned}
$$



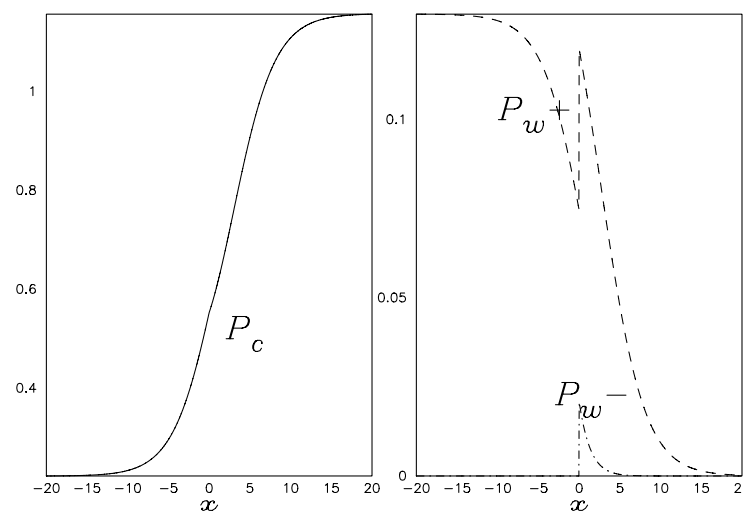

Fig. 2. The cosmic ray and Alfvén wave pressures for shocked background (Ko 1998). In this example, $\gamma_{\mathrm{c}}=\frac{4}{3}, \beta=\frac{4}{3}$, the upstream Alfvénic Mach number is $M_{\mathrm{A} 1}=2.0$, the compression ratio is $q=1.5$, and the location is $\sigma=0.6$.

By substituting the steady state profiles of Sect. 3 into the linearised equations and consider some suitable boundary conditions, one will obtain an eigenvalue problem. In this work, we consider short-length scale perturbations (short compared to the variation of the unperturbed profiles). We therefore approximate the perturbations as $\delta \psi \propto \exp [\mathrm{i}(k x-\omega t)]$ and neglect the gradients of the unperturbed profiles, obtaining

$\left[\begin{array}{lll}A_{\mathrm{cc}} & A_{\mathrm{c}+} & A_{\mathrm{c}-} \\ A_{+\mathrm{c}} & A_{++} & A_{+-} \\ A_{-\mathrm{c}} & A_{-+} & A_{--}\end{array}\right]\left[\begin{array}{l}\delta P_{\mathrm{c}} \\ \delta P_{\mathrm{w}}^{+} \\ \delta P_{\mathrm{w}}^{-}\end{array}\right]=0$,

where

$$
\begin{aligned}
A_{\mathrm{cc}}= & -\mathrm{i} \omega+\mathrm{i}\left[U+\left(e_{+}-e_{-}\right) V_{\mathrm{A}}\right] k+\frac{c^{2} k^{2}}{3 \alpha\left(P_{\mathrm{w}}^{+}+P_{\mathrm{w}}^{-}\right)} \\
& -16 \alpha\left(\gamma_{\mathrm{c}}-1\right) \frac{V_{\mathrm{A}}^{2}}{c^{2}} e_{+} e_{-}\left(P_{\mathrm{w}}^{+}+P_{\mathrm{w}}^{-}\right), \\
A_{\mathrm{c}+}= & \mathrm{i} 2 \gamma_{\mathrm{c}} e_{-} e_{\mathrm{c}} V_{\mathrm{A}} k-16 \alpha\left(\gamma_{\mathrm{c}}-1\right) \frac{V_{\mathrm{A}}^{2}}{c^{2}} e_{-}^{2} P_{\mathrm{c}}, \\
A_{\mathrm{c}-}= & -\mathrm{i} 2 \gamma_{\mathrm{c}} e_{+} e_{\mathrm{c}} V_{\mathrm{A}} k-16 \alpha\left(\gamma_{\mathrm{c}}-1\right) \frac{V_{\mathrm{A}}^{2}}{c^{2}} e_{+}^{2} P_{\mathrm{c}}, \\
A_{+\mathrm{c}}= & \mathrm{i} \frac{1}{2} e_{+} V_{\mathrm{A}} k+4 \alpha \frac{V_{\mathrm{A}}^{2}}{c^{2}} e_{+} e_{-}\left(P_{\mathrm{w}}^{+}+P_{\mathrm{w}}^{-}\right), \\
A_{++}= & -\mathrm{i} \omega+\mathrm{i}\left(U+V_{\mathrm{A}}\right) k+4 \alpha \frac{V_{\mathrm{A}}^{2}}{c^{2}} e_{-}^{2} P_{\mathrm{c}}, \\
A_{+-}= & 4 \alpha \frac{V_{\mathrm{A}}^{2}}{c^{2}} e_{+}^{2} P_{\mathrm{c}}, \\
A_{-\mathrm{c}}= & -\mathrm{i} \frac{1}{2} e_{-} V_{\mathrm{A}} k+4 \alpha \frac{V_{\mathrm{A}}^{2}}{c^{2}} e_{+} e_{-}\left(P_{\mathrm{w}}^{+}+P_{\mathrm{w}}^{-}\right), \\
A_{-+}= & 4 \alpha \frac{V_{\mathrm{A}}^{2}}{c^{2}} e_{-}^{2} P_{\mathrm{c}}, \\
A_{--}= & -\mathrm{i} \omega+\mathrm{i}\left(U-V_{\mathrm{A}}\right) k+4 \alpha \frac{V_{\mathrm{A}}^{2}}{c^{2}} e_{+}^{2} P_{\mathrm{c}} .
\end{aligned}
$$

Nontrivial solutions for $\left(\delta P_{\mathrm{c}}, \delta P_{\mathrm{w}}^{ \pm}\right)$require the vanishing of the determinant of the matrix above and give us the dispersion relation. To make the final expression of the dispersion relation neater, we define the normalised frequency and wavenumber as,

$\tilde{\omega}=(\omega-U k) l_{\mathrm{D}} / V_{\mathrm{A}}, \quad \tilde{k}=k l_{\mathrm{D}}$ and

$$
l_{\mathrm{D}}=\frac{c^{2}}{3 \alpha V_{\mathrm{A}}\left(P_{\mathrm{w}}^{+}+P_{\mathrm{w}}^{-}\right)},
$$

is the diffusion length scale. After some manipulation, we obtain the dispersion relation,

$$
\begin{aligned}
0= & \tilde{\omega}^{3}+\tilde{\omega}^{2}\left[a_{2} \tilde{k}+\mathrm{i}\left(b_{2}+\tilde{k}^{2}\right)\right]-\tilde{\omega} \tilde{k}\left(a_{1} \tilde{k}+\mathrm{i} b_{1}\right) \\
& -\tilde{k}^{2}\left[\left(a_{2}+a_{0}\right) \tilde{k}+\mathrm{i}\left(b_{0}+\tilde{k}^{2}\right)\right],
\end{aligned}
$$

where

$a_{2}=e_{-}-e_{+}$,

$a_{1}=1+2 \gamma_{\mathrm{c}} e_{\mathrm{c}} e_{+} e_{-}+\frac{4}{3} e_{\mathrm{c}}\left(e_{+}^{2}+e_{-}^{2}\right)$,

$a_{0}=\frac{4}{3} e_{\mathrm{c}}\left(e_{-}-e_{+}\right)$,

$b_{2}=\frac{4}{3}\left[e_{\mathrm{c}}\left(e_{+}^{2}+e_{-}^{2}\right)-4\left(\gamma_{\mathrm{c}}-1\right) e_{+} e_{-}\right]$,

$b_{1}=-\frac{8}{3} e_{\mathrm{c}}\left(e_{-}-e_{+}\right)$,

$b_{0}=-\frac{4}{3}\left[e_{\mathrm{c}}\left(e_{+}^{2}+e_{-}^{2}\right)+4\left(\gamma_{\mathrm{c}}-1\right) e_{+} e_{-}-\gamma_{\mathrm{c}} e_{\mathrm{c}}^{2} e_{+} e_{-}\right]$,

and $e_{\mathrm{c}}=P_{\mathrm{c}} /\left(P_{\mathrm{w}}^{+}+P_{\mathrm{w}}^{-}\right)$. We normalise the frequency to the diffusion time scale $l_{\mathrm{D}} / V_{\mathrm{A}}$. This is a natural time-scale of our system, but it depends on $\left(P_{\mathrm{w}}^{+}+P_{\mathrm{w}}^{-}\right)$which varies with position. However, this will not affect the stability criteria of the system. Another choice is the time scale $1 / V_{\mathrm{A}} k$, but it is not convenient for later discussions on the dispersion relation in the limits of large or small $k$.

Finally, we note that the dispersion relation Eq. (29) is invariant if we exchange $P_{\mathrm{w}}^{+}$and $P_{\mathrm{w}}^{-}$, and change $k$ to $-k$.

\section{Results}

In general, Eq. (29) has three complex roots for $\tilde{\omega}$. If the imaginary part of $\omega$ is positive (i.e., $\omega_{\mathrm{i}}>0$ which is the same as $\tilde{\omega}_{\mathrm{i}}>0$ ), the system is unstable (at least in the linear stage). We present some limiting cases before we go to the general cases.

\subsection{Limiting cases}

It is possible to express $\tilde{\omega}_{\mathrm{i}}>0$ of some limiting cases analytically. Here we show the cases: (i) $P_{\mathrm{w}}^{-} \ll P_{\mathrm{w}}^{+}$or $P_{\mathrm{w}}^{+} \ll P_{\mathrm{w}}^{-}$; (ii) $P_{\mathrm{c}} \gg\left(P_{\mathrm{w}}^{+}+P_{\mathrm{w}}^{-}\right)$; (iii) $P_{\mathrm{c}} \ll\left(P_{\mathrm{w}}^{+}+P_{\mathrm{w}}^{+}\right)$; (iv) $k l_{\mathrm{D}} \gg 1$; and (v) $k l_{\mathrm{D}} \ll 1$.

\subsection{1. $P_{\mathrm{w}}^{-} \ll P_{\mathrm{w}}^{+}$or $P_{\mathrm{w}}^{+} \ll P_{\mathrm{w}}^{-}$}

First, for the case of uni-directional wave systems, (i.e., either a forward-wave system $\left(e_{+}, e_{-}\right)=(1,0)$ or a backward-wave system $\left.\left(e_{+}, e_{-}\right)=(0,1)\right)$, see upper panel of Fig. 1$)$, it can easily be shown that

$\tilde{\omega}= \pm \tilde{k}, \quad \pm \tilde{k}-\mathrm{i} \tilde{k}^{2}, \quad \mp \tilde{k}-\mathrm{i} \frac{4}{3} e_{\mathrm{c}}$,

where the upper and lower signs correspond to forward- and backward-wave systems, respectively. Two of them have negative imaginary parts and one of them is real, thus uni-directional wave systems are unconditionally stable in this approximation. (Please note that if we put in the gradients of the unperturbed profile the systems become unstable.)

Now let us consider quasi-uni-directional wave systems, i.e., both waves exist, but one of them is very small $\left(e_{-} \ll 1\right.$ or $\left.e_{+} \ll 1\right)$. Small $e_{-}$or $e_{+}$may give rise to some extra imaginary part of the roots, thus we should focus on the real root $\pm \tilde{k}$, 


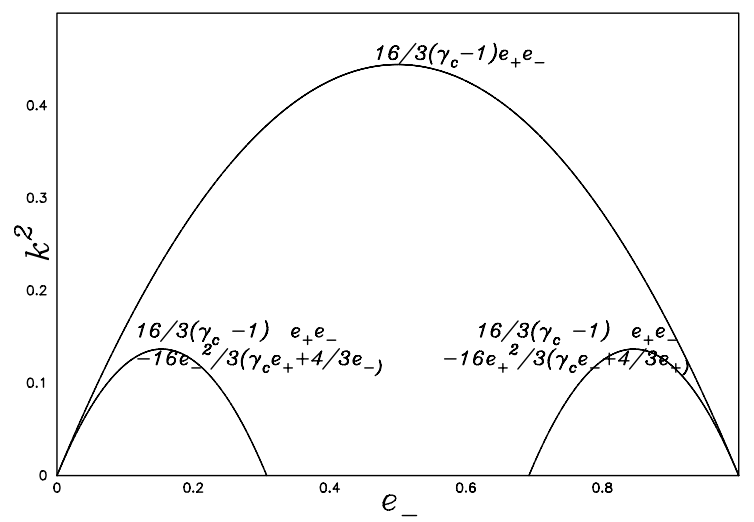

Fig. 3. When $P_{\mathrm{c}}$ tends to zero (i.e., $\left.P_{\mathrm{c}} \ll\left(P_{\mathrm{w}}^{+}+P_{\mathrm{w}}^{-}\right)\right)$, the system is unstable in the region under the curve (i.e., $\tilde{k}^{2}=\frac{16}{3}\left(\gamma_{\mathrm{c}}-1\right) e_{+} e_{-}$, where $\left.e_{+}+e_{-}=1\right) \cdot \gamma_{\mathrm{c}}=\frac{4}{3}$ in this example.

because even a small extra positive imaginary part would make the system unstable. Substituting $\tilde{\omega}= \pm \tilde{k}+e_{\mp} \Omega_{1}$ into Eq. (29), we obtain

$\Omega_{1}=-\mathrm{i} \gamma_{\mathrm{c}} e_{\mathrm{c}}$.

Therefore the system is stable up to first order in $e_{-}$or $e_{+}$.

\subsection{2. $P_{\mathrm{c}} \gg\left(P_{\mathrm{w}}^{+}+P_{\mathrm{w}}^{-}\right)$}

Figure 1 shows that $P_{\mathrm{w}}^{ \pm}$decay to zero while $P_{\mathrm{c}}$ remain finite as $x \rightarrow \infty$. Thus $P_{\mathrm{c}} \gg\left(P_{\mathrm{w}}^{+}+P_{\mathrm{w}}^{-}\right)$and $e_{\mathrm{c}} \gg 1$. It turns out that when $e_{\mathrm{c}} \gg 1$, one of the roots of Eq. (29) is of the order of $e_{\mathrm{c}}$. It is more convenient to define a new frequency $\tilde{\omega}_{\mathrm{c}}=\tilde{\omega} / e_{\mathrm{c}}$. To the zeroth order of $1 / e_{\mathrm{c}}$, the roots of Eq. (29) are

$\tilde{\omega}_{\mathrm{c}}=0, \quad 0, \quad-\mathrm{i} \frac{4}{3}\left(e_{+}^{2}+e_{-}^{2}\right)$.

The system is stable in this limit. Now focus on the real roots 0 , and let $\tilde{\omega}_{\mathrm{c}}=\Omega_{1} / \sqrt{e_{\mathrm{c}}}+\Omega_{2} / e_{\mathrm{c}}$ (it turns out that the next order term is $e_{\mathrm{c}}^{-1 / 2}$ which is real, and we must consider the next higher order term to examine the stability). We obtain

$\Omega_{1}= \pm \sqrt{\frac{\gamma_{\mathrm{c}} e_{+} e_{-}}{\left(e_{+}^{2}+e_{-}^{2}\right)}} \tilde{k}$,

and

$\Omega_{2}=\frac{\left(e_{+}-e_{-}\right)}{\left(e_{+}^{2}+e_{-}^{2}\right)} \tilde{k}-\mathrm{i}\left[\frac{1}{2}+\frac{3 \gamma_{\mathrm{c}} e_{+} e_{-}\left(1-4 e_{+} e_{-}\right)}{8\left(e_{+}^{2}+e_{-}^{2}\right)^{2}}\right]$.

Since $4 e_{+} e_{-}=\left(e_{+}+e_{-}\right)^{2}-\left(e_{+}-e_{-}\right)^{2}=1-\left(e_{+}-e_{-}\right)^{2} \leq 1$, the system is stable up to $e_{\mathrm{c}}^{-1}$ for large $e_{\mathrm{c}}$.

\subsection{3. $P_{\mathrm{c}} \ll\left(P_{\mathrm{w}}^{+}+P_{\mathrm{w}}^{-}\right)$}

Figure 1 shows that $P_{\mathrm{c}}$ tends to zero in the far upstream region $(x \rightarrow-\infty)$. Thus $P_{\mathrm{c}} \ll\left(P_{\mathrm{w}}^{+}+P_{\mathrm{w}}^{-}\right)$and $e_{\mathrm{c}} \ll 1$. Setting $e_{\mathrm{c}}=0$ in Eq. (29) gives

$\tilde{\omega}= \pm \tilde{k}, \quad\left(e_{+}-e_{-}\right) \tilde{k}+\mathrm{i}\left[\frac{16}{3}\left(\gamma_{\mathrm{c}}-1\right) e_{+} e_{-}-\tilde{k}^{2}\right]$.

$\mathfrak{J}(\tilde{\omega})>0$ if

$\tilde{k}^{2}<\frac{16}{3}\left(\gamma_{\mathrm{c}}-1\right) e_{+} e_{-}$.

When $\tilde{k}$ is small enough the system is unstable in this limit. The curve in Fig. 3 is $\tilde{k}^{2}=\frac{16}{3}\left(\gamma_{\mathrm{c}}-1\right) e_{+} e_{-}$, under which the system is unstable.
Now let us focus on the real roots $\pm \tilde{k}$, and let $\tilde{\omega}= \pm \tilde{k}+e_{\mathrm{c}} \Omega_{1}$.

$$
\begin{aligned}
& \Omega_{1}=\frac{e_{\mp} \tilde{k}\left[\left(\frac{4}{3} e_{\mp}+\gamma_{\mathrm{c}} e_{ \pm}\right) \tilde{k} \mp \mathrm{i} \frac{8}{3} e_{\mp}\right]}{\left\{ \pm 2 e_{\mp} \tilde{k}+i\left[\tilde{k}^{2}-\frac{16}{3}\left(\gamma_{\mathrm{c}}-1\right) e_{+} e_{-}\right]\right\}} \\
& \mathfrak{J}\left(\Omega_{1}\right)>0 \text { if } \\
& \tilde{k}^{2}<\frac{16}{3}\left(\gamma_{\mathrm{c}}-1\right) e_{+} e_{-}-\frac{16 e_{\mp}^{2}}{3\left(\gamma_{\mathrm{c}} e_{ \pm}+\frac{4}{3} e_{\mp}\right)} \\
& <\frac{16}{3}\left(\gamma_{\mathrm{c}}-1\right) e_{+} e_{-} .
\end{aligned}
$$

Thus the unstable region is already within the region described by Eq. (37).

\subsection{4. $k l_{D} \gg 1$}

We now turn to the parameter of the perturbations, say the wavelength of the perturbations. When the wavelength is very short, it turns out that one of the roots of Eq. (29) is of the order of $\tilde{k}^{2}$. In this case it is more convenient to define a new frequency $\tilde{\omega}_{k}=\tilde{\omega} / \tilde{k}^{2}$. To the zeroth order of $1 / \tilde{k}$, the roots of Eq. (29) are

$\tilde{\omega}_{k}=0, \quad 0, \quad-\mathrm{i}$.

The system is stable in this limit. Now focus on the real roots 0 ; if we let $\tilde{\omega}_{k}=\Omega_{1} / \tilde{k}+\Omega_{2} / \tilde{k}^{2}$, then we obtain

$\Omega_{1}= \pm 1$,

$\Omega_{2}=-\mathrm{i}\left(\gamma_{\mathrm{c}} e_{\mathrm{c}} e_{+} e_{-}+\frac{4}{3} e_{\mathrm{c}} e_{\mp}^{2}\right)$.

The system is unconditionally stable up to the second order in $\tilde{k}^{-2}$. In fact, when we solve Eq. (29) numerically, we find that the system is stable for large enough $\tilde{k}$.

\subsection{5. $k l_{\mathrm{D}} \ll 1$}

For completeness we include a discussion on the case of long wavelength perturbations. (This is just for academic interest as the analysis in Sect. 4 may not be valid in the long wavelength limit for the steady state in Sect. 3.) When $\tilde{k}=0$, the roots of Eq. (29) are

$\tilde{\omega}=0, \quad 0, \quad \mathrm{i} \frac{4}{3}\left[4\left(\gamma_{\mathrm{c}}-1\right) e_{+} e_{-}-e_{\mathrm{c}}\left(e_{+}^{2}+e_{-}^{2}\right)\right]$.

The dashed-line in Fig. 4 is $e_{\mathrm{c}}=4 e_{+} e_{-} /\left(e_{+}^{2}+e_{-}^{2}\right)$, i.e., $b_{2}=0$. In this limit, the system is unstable when $b_{2}<0$ (i.e., under the dashed-line in Fig. 4). For $b_{2}>0$, we should focus on the real roots 0 to examine stability. Let $\tilde{\omega}=\tilde{k} \Omega_{1}+\tilde{k}^{2} \Omega_{2}$, (it turns out that the first order term can be real, and we must consider the next higher order term). After some manipulations we find that $\Omega_{1}$ and $\Omega_{2}$ satisfy

$b_{2} \Omega_{1}^{2}-b_{1} \Omega_{1}-b_{0}=0$

and

$\Omega_{2}=\frac{\mathrm{i}}{2} \frac{\mathcal{N}}{\mathcal{D}}=\frac{\mathrm{i}}{2} \frac{\left[\Omega_{1}^{3}+a_{2} \Omega_{1}^{2}-a_{1} \Omega_{1}-\left(a_{0}+a 2\right)\right]}{\left(b_{2} \Omega_{1}-\frac{1}{2} b_{1}\right)}$.

If $b_{1}^{2}+4 b_{0} b_{2}<0$, the two roots of Eq. (44) are complex conjugates, thus the imaginary part of one of the roots is positive, and the system is unstable. This corresponds to the region bounded by the dotted-line in Fig. 4 . If $b_{1}^{2}+4 b_{0} b_{2}>0, \Omega_{1}$ is 


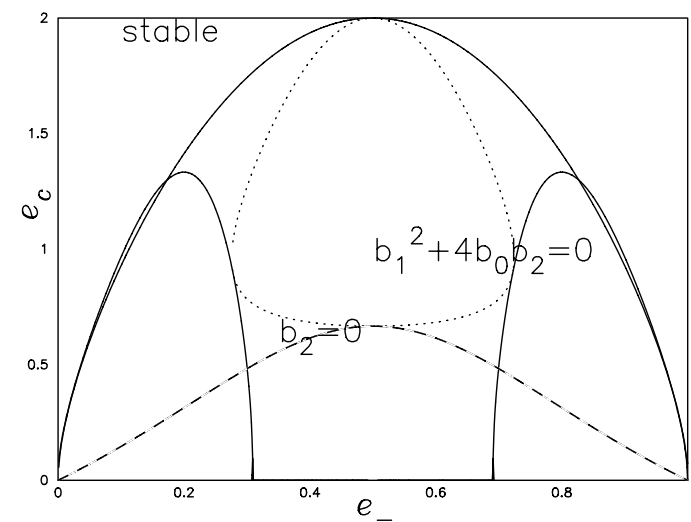

Fig. 4. When $k$ tends to zero (i.e., $k l_{\mathrm{D}} \ll 1$ ), the system is unstable in the regions under the solid-line (given by $\mathcal{A}_{0} \mathcal{C}_{0}-\mathcal{B}_{0}^{2}=0$ ). The dashed-line is given by $b_{2}=0$ and the dotted-line by $b_{1}^{2}+4 b_{0} b_{2}=0 . \gamma_{\mathrm{c}}=\frac{4}{3}$ in this example.

real and we must study $\Omega_{2}$. Since $\Omega_{2}$ is purely imaginary, the system is unstable if $\Omega_{2}>0$. Elimination of $\Omega_{1}$ from $\mathcal{N}=0$ (i.e., $\Omega_{1}^{3}+a_{2} \Omega_{1}^{2}-a_{1} \Omega_{1}-a_{0}-a_{2}=0$ ) and Eq. (44) (i.e., $\left.b_{2} \Omega_{1}^{2}-b_{1} \Omega_{1}-b_{0}=0\right)$ gives the marginally stable condition,

$\mathcal{A}_{0} C_{0}-\mathcal{B}_{0}^{2}=0$,

where

$$
\begin{aligned}
\mathcal{A}_{0} & =b_{1} b_{2}\left(a_{0}+a_{2}\right)-b_{0}\left(a_{1} b_{2}-b_{0}\right), \\
\mathcal{B}_{0} & =b_{0}\left(a_{2} b_{2}+b_{1}\right)-b_{2}^{2}\left(a_{0}+a_{2}\right), \\
C_{0} & =b_{1}\left(a_{2} b_{2}+b_{1}\right)-b_{2}\left(a_{1} b_{2}-b_{0}\right),
\end{aligned}
$$

and $a_{j}, b_{j}(j=0,1,2)$ are given by Eq. (30). This result is reminiscent of the general result using Hermite-Biehler theorem (see Sect. 5.2). This unstable region is located under the solid-line in Fig. 4.

As a whole, the system is unstable in the limit $k l_{\mathrm{D}} \ll 1$ when the parameters are under the solid-line in Fig. 4 (given by Eq. (46)), because the region includes the regions bounded by the dashed- and dotted-lines individually.

\subsection{General case}

From the limiting cases we understand that the system is stable for short wavelength perturbations, when the cosmic ray energy density is large, and the wave energy densities (at least one of the two) is small. In fact, the general stability region in the parameter space can be obtained by the Hermite-Biehler theorem (see e.g., Bhattacharyya et al. 1995). We shall not go into the detail of the theorem, but rather just present the result. For marginally stable cases the condition can be obtained by eliminating $\Omega$ from the following two equations,

$\Omega^{3}+a_{2} \tilde{k} \Omega^{2}-a_{1} \tilde{k}^{2} \Omega-\left(a_{0}+a_{2}\right) \tilde{k}^{3}=0$,

and

$\left(b_{2}+\tilde{k}^{2}\right) \Omega^{2}-b_{1} \tilde{k} \Omega-\left(b_{0}+\tilde{k}^{2}\right) \tilde{k}^{2}=0$.

Explicitly, the condition is

$\mathcal{A C}-\mathcal{B}^{2}=0$, where

$$
\begin{aligned}
\mathcal{A}= & b_{1}\left(b_{2}+\tilde{k}^{2}\right)\left(a_{0}+a_{2}\right) \\
& -\left(b_{0}+\tilde{k}^{2}\right)\left[a_{1}\left(b_{2}+\tilde{k}^{2}\right)-\left(b_{0}+\tilde{k}^{2}\right)\right], \\
\mathcal{B}= & \left(b_{0}+\tilde{k}^{2}\right)\left[a_{2}\left(b_{2}+\tilde{k}^{2}\right)+b_{1}\right] \\
& -\left(b_{2}+\tilde{k}^{2}\right)^{2}\left(a_{0}+a_{2}\right), \\
\mathcal{C}= & b_{1}\left[a_{2}\left(b_{2}+\tilde{k}^{2}\right)+b_{1}\right] \\
& -\left(b_{2}+\tilde{k}^{2}\right)\left[a_{1}\left(b_{2}+\tilde{k}^{2}\right)-\left(b_{0}+\tilde{k}^{2}\right)\right],
\end{aligned}
$$

and $a_{j}, b_{j}(j=0,1,2)$ are given by Eq. (30). In fact, the lefthand side of Eq. (50) can be factored as $\mathcal{A C}-\mathcal{B}^{2}=e_{+} e_{-} e_{\mathrm{c}}^{2}\left(b_{2}+\right.$ $\left.\tilde{k}^{2}\right) \mathcal{H}$, where $\mathcal{H}$ is a complicated polynomial in $\left(\gamma_{\mathrm{c}}, e_{+}, e_{-}, e_{\mathrm{c}}, \tilde{k}\right)$. When we express $e_{+}=1-e_{-}$, the highest degree of $\gamma_{\mathrm{c}}$ in $\mathcal{H}$ is 5 , of $e_{-}$is 8 , of $e_{\mathrm{c}}$ is 4 , and of $\tilde{k}$ is 6 . Figure 5 shows the unstable region in the parameter space $\left(e_{-}, e_{\mathrm{c}}\right)$ for different $\tilde{k}$. The isosurface corresponding to $\mathcal{H}=0$ is shown in Fig. 6 .

The analysis above gives us the criteria on the (linear) stability of the system, but it cannot provide a quantitative comparison of the growth rates. To illustrate ideas, we take the steady state discussed in Sect. 3 as an example. Since the diffusion length scale $l_{\mathrm{D}}$ (see Eq. (28)) differs at different locations along the flow $\left(P_{\mathrm{w}}^{+}\right.$and $P_{\mathrm{w}}^{-}$vary at different $x$, see Fig. 1$)$, the time scale $l_{\mathrm{D}} / V_{\mathrm{A}}$ changes accordingly. In order to compare the growth rates at different locations we better normalised them to one and only one time scale. Instead of $\tilde{\omega}$ and $\tilde{k}$ in Eq. (29), we use

$\bar{\omega}=\tilde{\omega} \frac{l_{\mathrm{D} 0}}{l_{\mathrm{D}}}, \quad \bar{k}=\tilde{k} \frac{l_{\mathrm{D} 0}}{l_{\mathrm{D}}}$,

where $l_{\mathrm{D} 0}$ is $l_{\mathrm{D}}$ at a particular location.

As shown in Sect. 5.1.1, the uni-directional wave systems are stable, hence we examine the two-wave systems only. We pick several locations of the steady-state solutions in the lower panels of Fig. 1. From upstream to downstream, for solution (d) they are: $x=-20,-10,0,5,10,15,20$; for (e) $x=-24,-12$, $0,6,12,18,21$; and for (f) $x=-20,0,5,10,15,20$. These locations are marked by " $\odot$ plus number" in the top panels of Fig. 7. The middle panels of Fig. 7 are the dispersion relations $(\mathfrak{J}(\bar{\omega})$ against $\bar{k}$ ) in the upstream regions (corresponding to numbers 1 to 4 in the top panels), and the bottom panels are the dispersion relations $(\mathfrak{J}(\bar{\omega})$ against $\bar{k}$ ) (corresponding to numbers 5 to 7 in the top panels).

For large enough $\bar{k}$ the system is stable everywhere (see Sect. 5.1.4). For smaller $\bar{k}$, when we move from upstream to downstream regions, the system changes from unstable to less unstable to stable in downstream regions. The steady-state solutions of the two-wave system discussed in Sect. 3 are unstable upstream and stable downstream.

In the case of shocked background, the system is stable in the upstream of the shock if only one wave exists there (see Eq. (31)). To simplify discussion, we consider unidirectional wave systems upstream, specifically, we consider $P_{\mathrm{w}}^{-}=0$ upstream. Figure 2 shows a possible background profile. Both waves occur downstream because the shock generated backward waves and amplified the forward wave, and therefore may be susceptible to the instability described above. Both waves decrease further downstream while cosmic ray pressure continues to increase (see other possible profiles in Ko 1998). In particular, the quantity $e_{\mathrm{c}}=P_{\mathrm{c}} /\left(P_{\mathrm{w}}^{+}+P_{\mathrm{w}}^{-}\right)$increases downstream. From the analysis above, we notice that the system is stable if $e_{\mathrm{c}}$ is large enough (in the case of Fig. 5 the system is stable if $e_{\mathrm{c}}>2$ ). Hence, if the system is stable just downstream of the shock it is stable throughout the downstream region. 

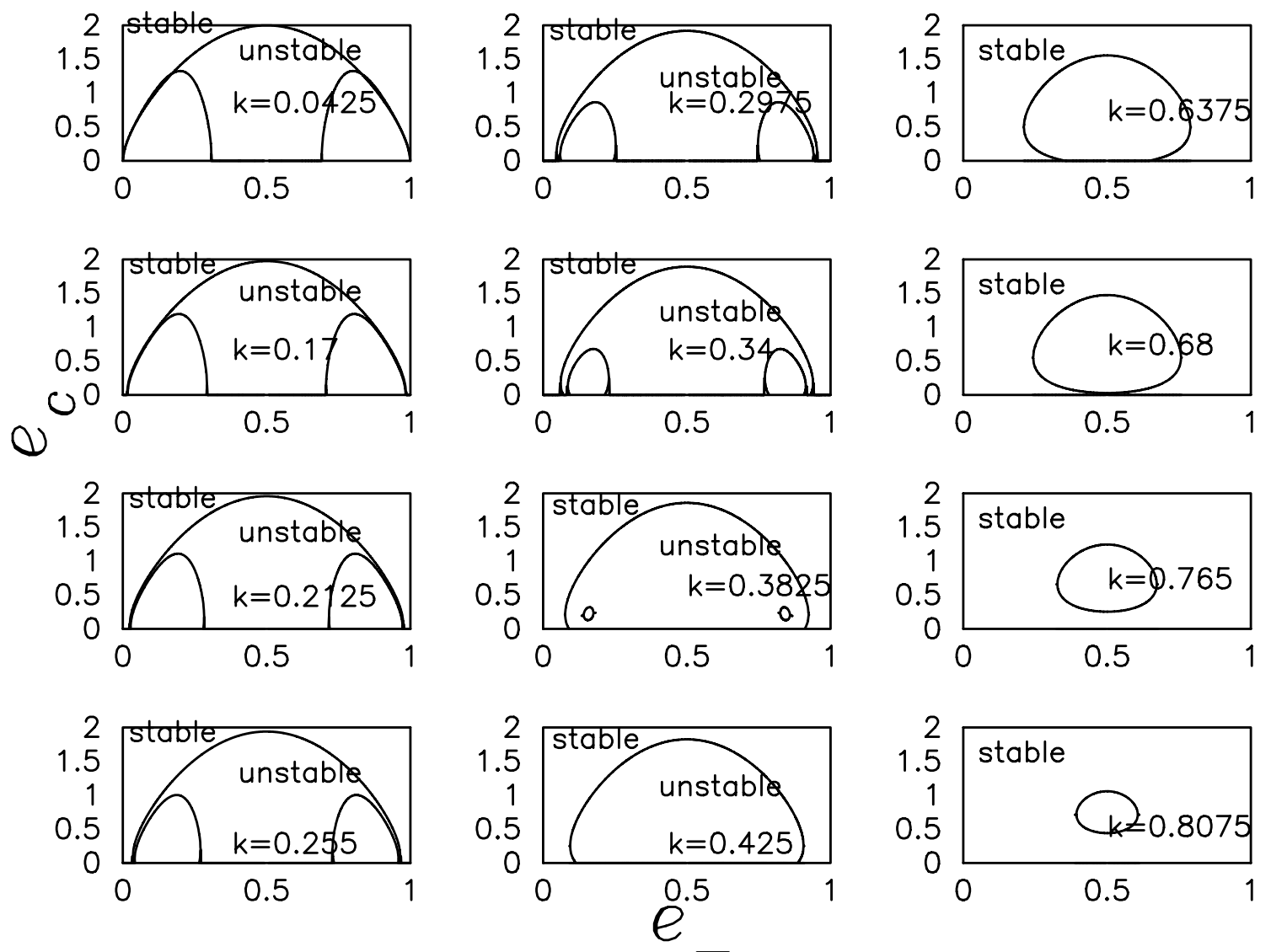

Fig. 5. The linearly unstable region of the system in the parameter space $\left(e_{-}, e_{\mathrm{c}}\right)$ for different $\tilde{k} . \gamma_{\mathrm{c}}=\frac{4}{3}$ in this example.

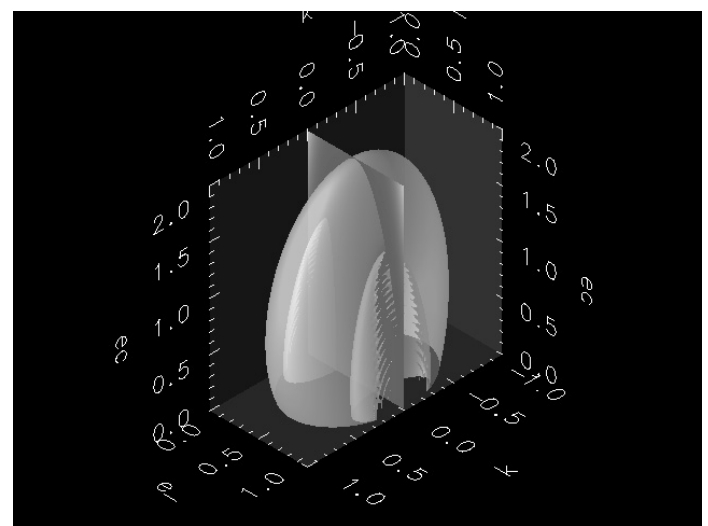

Fig. 6. The isosurface of $\mathcal{H}=0$ in parameter space $\left(e_{-}, e_{\mathrm{c}}, \tilde{k}\right) \cdot \gamma_{\mathrm{c}}=\frac{4}{3}$ in this example.

Figure 8 shows the stable region in the compression ratioshock location parameter space. Above the dashed line is a forbidden region because $P_{\mathrm{c}}<0$ there. The region below the solid line is a stable region. Depending on other parameters in the system, the region in-between may or may not be stable.

\section{Summary and discussion}

The hydrodynamic approach of cosmic ray propagation is a rather useful approximation for the structure and evolution of cosmic-ray-plasma systems. One-dimensional steady-state solutions at different sophisticated levels have been found (e.g.,
Drury \& Völk 1981; McKenzie \& Völk 1982; Axford et al. 1982; Jiang et al. 1996; Ko 1998; Ko 2001). The stability of some of these systems has been studied. For cosmicray-modified shocks without wave, they are most likely susceptible to the so-called squeezing instability (e.g., Drury \& Falle 1986; Zank \& McKenzie 1987; Zank et al. 1990; Kang et al. 1992; Zank et al. 1993). For systems with one wave, only uniform state has been analysed carefully (McKenzie \& Webb 1984; Zank 1989; Ko \& Jeng 1994). They are susceptible to the slower mode of the slow magneto-acoustic modes. (Note that although Ko \& Jeng 1994 allowed for two waves in their analysis, the unperturbed state was still uni-directional.) Owing to stochastic acceleration, the two-wave system is inherently nonuniform whether it is the full system (Ko 2001) or the simplified versions (Jiang et al. 1996; Ko 1998).

In this work, we study the stability of the steady-state solution of the simplified version of a two-wave system, in which the plasma flow is assumed to be constant and not affected by the cosmic rays and waves (Jiang et al. 1996). In this model the coupling between the constituents depends on the wave energy density or pressure. We perform linear perturbation analysis in short wavelength approximation (and neglect all the gradient of the unperturbed profile). We conclude that the system is stable in the short wavelength limit, and also stable in the limits of $P_{\mathrm{w}}^{-} \ll P_{\mathrm{w}}^{+}$, $P_{\mathrm{w}}^{+} \ll P_{\mathrm{w}}^{-}, P_{\mathrm{c}} \ll\left(P_{\mathrm{w}}^{+}+P_{\mathrm{w}}^{-}\right)$as well. However, it is susceptible to intermediate and long wavelength perturbations. Nevertheless, we must point out the present work is a short wavelength analysis, thus more detailed analysis is needed to substantiate the claim that the system is unstable in long wavelength limit. 

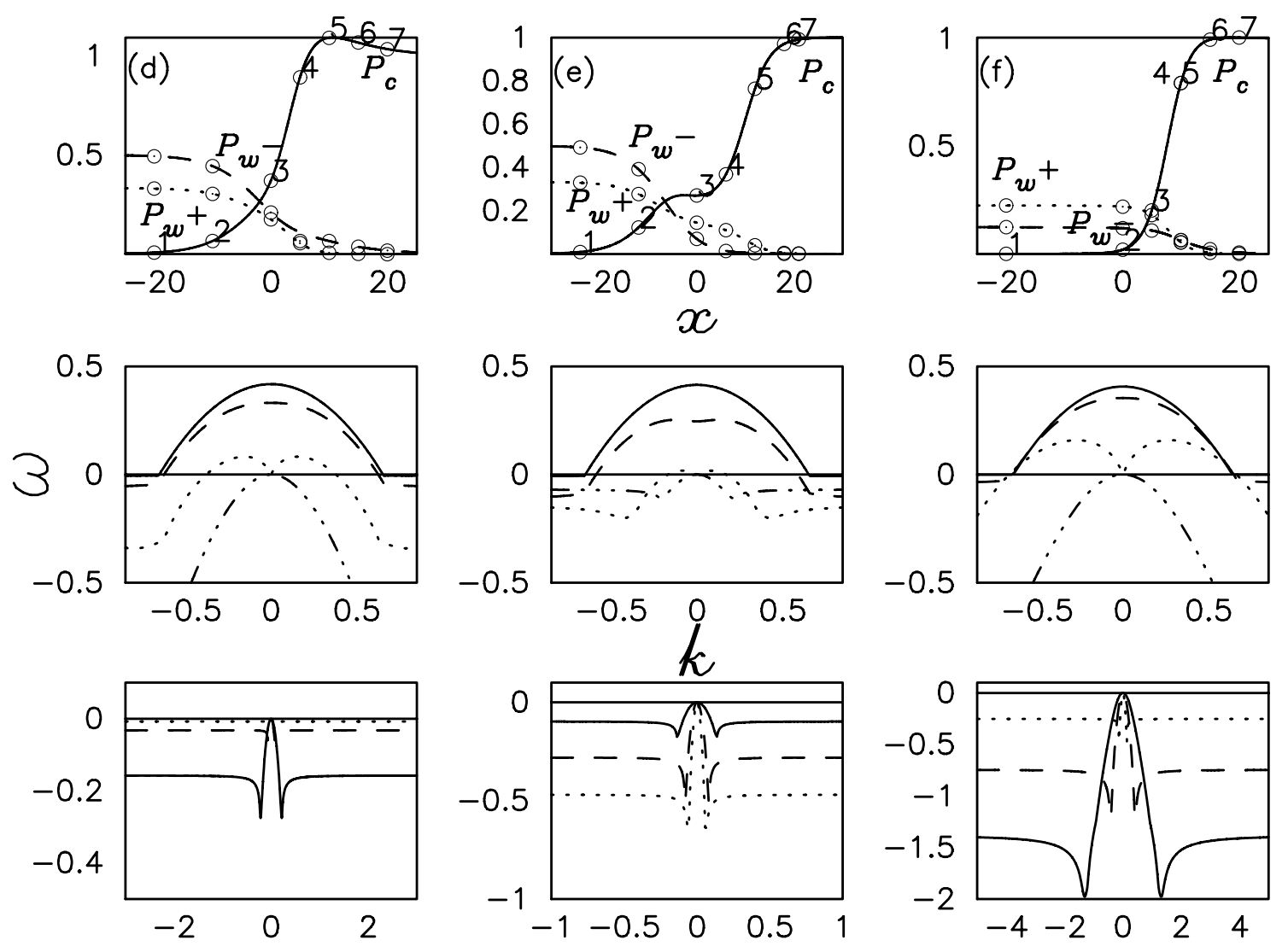

Fig. 7. Dispersion relations at different locations of three steady-state two-wave systems. The top panels show the steady-state profiles of Figs. 1d, e and $\mathrm{f}$. Location 1 to 7 are marked by $\odot$. For profile d) they are: $x=-20,-10,0,5,10,15$, 20; for e) $x=-24,-12,0,6,12,18,21$; and f) $x=-20,0,5,10,15,20$; respectively. The middle panels show the dispersion relations for upstream regions (numbers 1 to 4). The solid-, dashed-, dotted-, dotted-dashed-lines correspond to locations numbers 1, 2, 3 and 4, respectively. The bottom panels show the dispersion relations for downstream regions (numbers 5 to 7). The solid-, dashed-, dotted-lines correspond to locations number 5, 6 and 7, respectively.

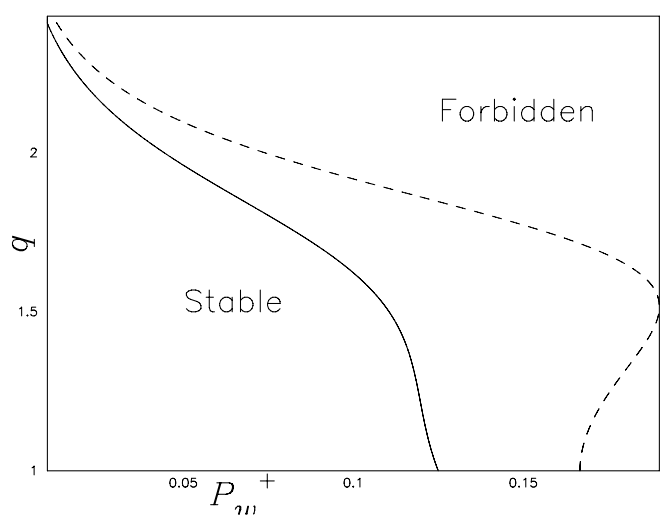

Fig. 8. Stable region in the parameter space $(q, \sigma)$ of the system in shocked background. The region above the dashed line is forbidden since $P_{c}<0$. The region below the solid line correspond to the stable region. The parameters for background are: $M_{\mathrm{A} 1}=2.0, P_{\mathrm{w}}^{+}=0.12$ far upstream.

The instability is caused by stochastic acceleration. If we deliberately take away the terms corresponding to stochastic acceleration the dispersion relation Eq. (29) becomes

$$
\begin{aligned}
0= & \tilde{\omega}^{3}+\tilde{\omega}^{2}\left[\mathrm{i} \tilde{k}^{2}+\tilde{k}\left(e_{-}-e_{+}\right)\right]-\tilde{\omega} \tilde{k}^{2}\left(1+2 \gamma_{\mathrm{c}} e_{\mathrm{c}} e_{+} e_{-}\right) \\
& -\tilde{k}^{2}\left[\mathrm{i} \tilde{k}^{2}+\tilde{k}\left(e_{-}-e_{+}\right)\right] .
\end{aligned}
$$

It can be shown (again by the Hermite-Biehler theorem) that all the roots have non-positive imaginary parts. That is, the system is stable.

In the case of uniform background flow, the system becomes stable far downstream. The reason is the unperturbed state tends to become a unidirectional wave system far downstream.

The present work does not take into account of the backreaction of cosmic rays and waves on the plasma flow. The analysis on the full system warrants further discussions.

Finally, the wave pressures we are discussing in this work are the average effect of an ensemble of short wavelength waves (generated by cosmic ray streaming instability and other mechanisms). In a way, they represent MHD turbulence. The large length scale instability presented in this work will certainly affect the structure of turbulence, and warrant further analysis.

Acknowledgements. This work is supported in part by the National Science Council of Taiwan, by grants NSC-94-2112-M-008-018 and NSC-95-2112-M008-006.

\section{References}

Achterberg, A., Blandford, R. D., \& Periwal, V. 1984, A\&A, 132, 97 Axford, W. I., Lee, E., \& McKenzie, J. F. 1982, A\&A, 111, 317

Berezhko, E. G., \& Krymskii, G. F. 1988, Soviet Phys. Usp., 31, 27

Bhattacharyya, S. P., Chapellat, H., \& Keel, L. H. 1995, Robust Control: The Parametric Approach (Prentice Hall)

Blandford, R. D., \& Eichler, D. 1987, Phys. Rep., 154, 1

Drury, L. O'C. 1983, Rep. Prog. Phys., 46, 973

Drury, L. O’C., \& Völk, H. J. 1981, ApJ, 248, 344

Drury, L. O'C., \& Falle, S. A. E. G. 1986, MNRAS, 223, 353 
Duffy, P., Drury, L. O’C., \& Völk, H. J. 1994, A\&A, 291, 613

Hanasz, M., \& Lesch, H. 2003, A\&A, 412, 331

Hanasz, M., Kowal, G., Otmianowska-Mazur, K., \& Lesch, H. 2004, ApJ, 605, L33

Heavens, A. F. 1984, MNRAS, 210, 813

Jiang, I. G., Chan, K. W., \& Ko, C. M. 1996, A\&A, 307, 903

Jones, T. W., \& Kang, H. 1990, ApJ, 363, 499

Jones, F. C., \& Ellison, D. C. 1991, Space Sci. Rev., 58, 259

Kang, H., Jones, T. W., \& Ryu, D. 1992, ApJ, 385, 193

Ko, C. M. 1991, A\&A, 242, 85

Ko, C. M. 1992, A\&A, 259, 377

Ko, C. M. 1995, Adv. Space Res., 15, 149

Ko, C. M. 1998, A\&A, 340, 605

Ko, C. M. 2001, J. Plasma Phys., 65, 305

Ko, C. M., \& Jeng, A. T. 1994, J. Plasma Phys., 52, 23

Ko, C. M., Doughetry, M. K., \& McKenize, J. F. 1991, A\&A, 241, 62

Ko, C. M., Chan, K. W., \& Webb, G. M. 1997, J. Plasma Phys., 57, 677

Kuwabara, T., \& Ko, C. M. 2006, ApJ, 636, 290

Kuwabara, T., Nakamura, K., \& Ko, C. M. 2004, ApJ, 607, 828
Kuznetsov, V. D., \& Ptuskin, V. S. 1983, Ap\&SS, 94, 5

Malkov, M. A. 1997a, ApJ, 485, 638

Malkov, M. A. 1997b, ApJ, 491, 584

Malkov, M. A., \& Drury, L. O'C. 2001, Rep. Prog. Phys., 64, 429

McKenzie, J. F., \& Völk, H. J. 1982, A\&A, 116, 191

McKenzie, J. F., \& Webb, G. M. 1984, J. Plasma Phys., 31, 275

Ptuskin, V. S. 1981, Ap\&SS, 76, 265

Ryu, D., Kim, J., Hong, S. S., \& Jones, T. W. 2003, ApJ, 589, 338

Schlickeiser, R. 1989, ApJ, 336, 243

Schlickeiser, R. 2002, Cosmic Ray Astrophysics (Springer: Berlin) Skilling, J. 1975a, MNRAS, 172, 557

Skilling, J. 1975b, MNRAS, 173, 255

Webb, G. M. 1987, ApJ, 319, 215

Webb, G. M., Drury, L. O'C., \& Völk, H. J. 1986, A\&A, 160, 335

Zank, G. P. 1989, J. Plasma Phys., 41, 89

Zank, G. P., \& Mckenzie, J. F. 1987, J. Plasma Phys., 37, 347

Zank, G. P., Axford, W. I., \& Mckenzie, J. F. 1990, A\&A, 233, 275

Zank, G. P., Webb, G. M., \& Donohue, D. J. 1993, ApJ, 406, 67 